\title{
Supplementary: Capturing The Iron Carburization Mechanisms From Surface To Bulk
}

El Tayeb Bentria ${ }^{a^{*}}$, Salawu Omotayo ${ }^{\mathrm{b}}$, Charlotte S. Becquart ${ }^{\mathrm{c}}$, Normand Mousseau ${ }^{\mathrm{d}}$, Othmane Bouhali ${ }^{\mathrm{b}}$ and Fedwa El-Mellouhi ${ }^{\mathrm{a}}$

Qatar Environment and Energy Research Institute, Hamad Bin Khalifa University, P.O. Box 34110, Doha, Qatar

Texas A\&M University at Qatar, P.O. Box 23874, Doha, Qatar

c Univ. Lille, CNRS, INRAE, Centrale Lille, UMR 8207 - UMET - Unité Matériaux et Transformations, F-59000 Lille,

France

d Département de physique and Regroupement québécois sur les matériaux de pointe, Université de Montréal, case postale 6128, succursale centre-ville, Montréal (QC) Canada H3C 3J7.

\section{Email : felmellouhi@hbku.edu.qa}

\section{Extended ReaxFF.}
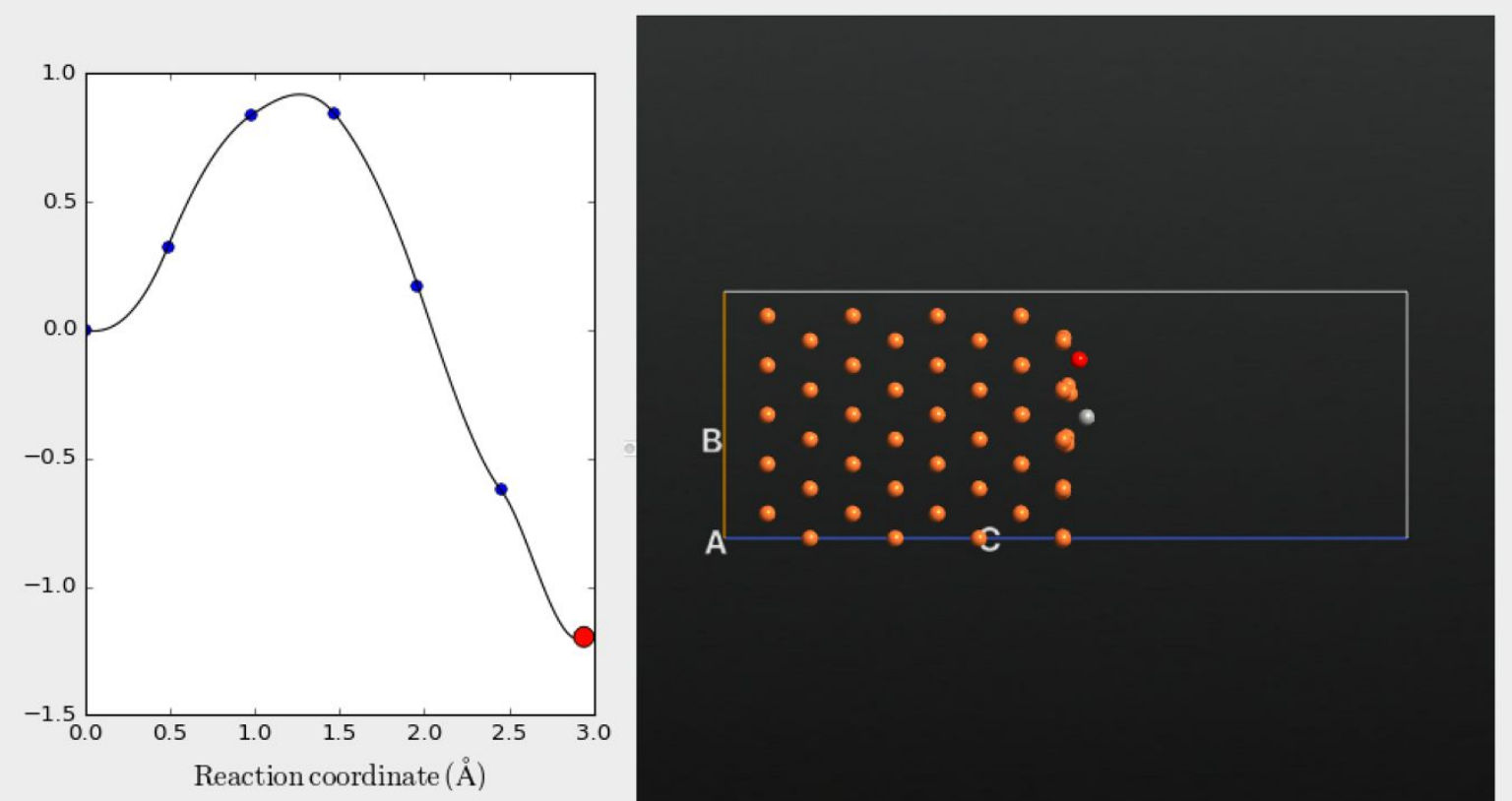

Figure S1: (left) Nudged elastic band diagram showing the dissociation barrier of CO molecule adsorbed at threefold position on iron (110) surface using Extended ReaxFF. (right) Ball and stick atomic representation of the final $\mathrm{CO}$ dissociated state iron (orange), oxygen (red) and carbon (gray). 


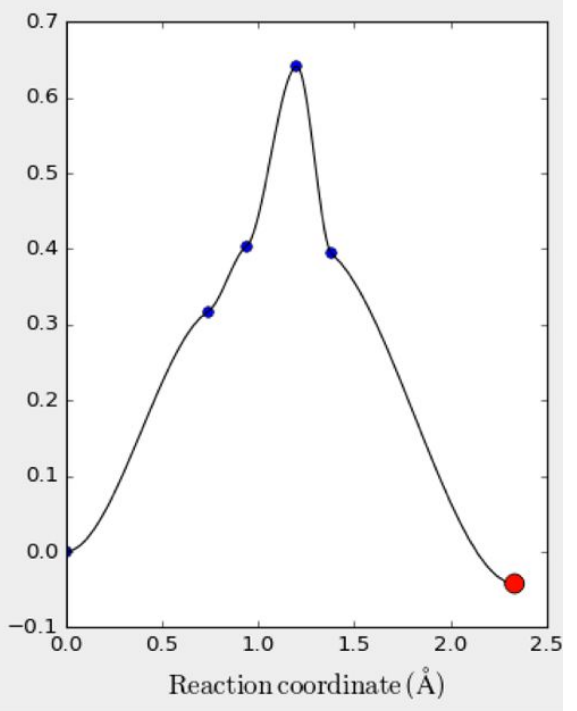

Figure S2: Nudged elastic band diagram of carbon diffusion in bulk iron through octahedral site using the extended ReaxFF.

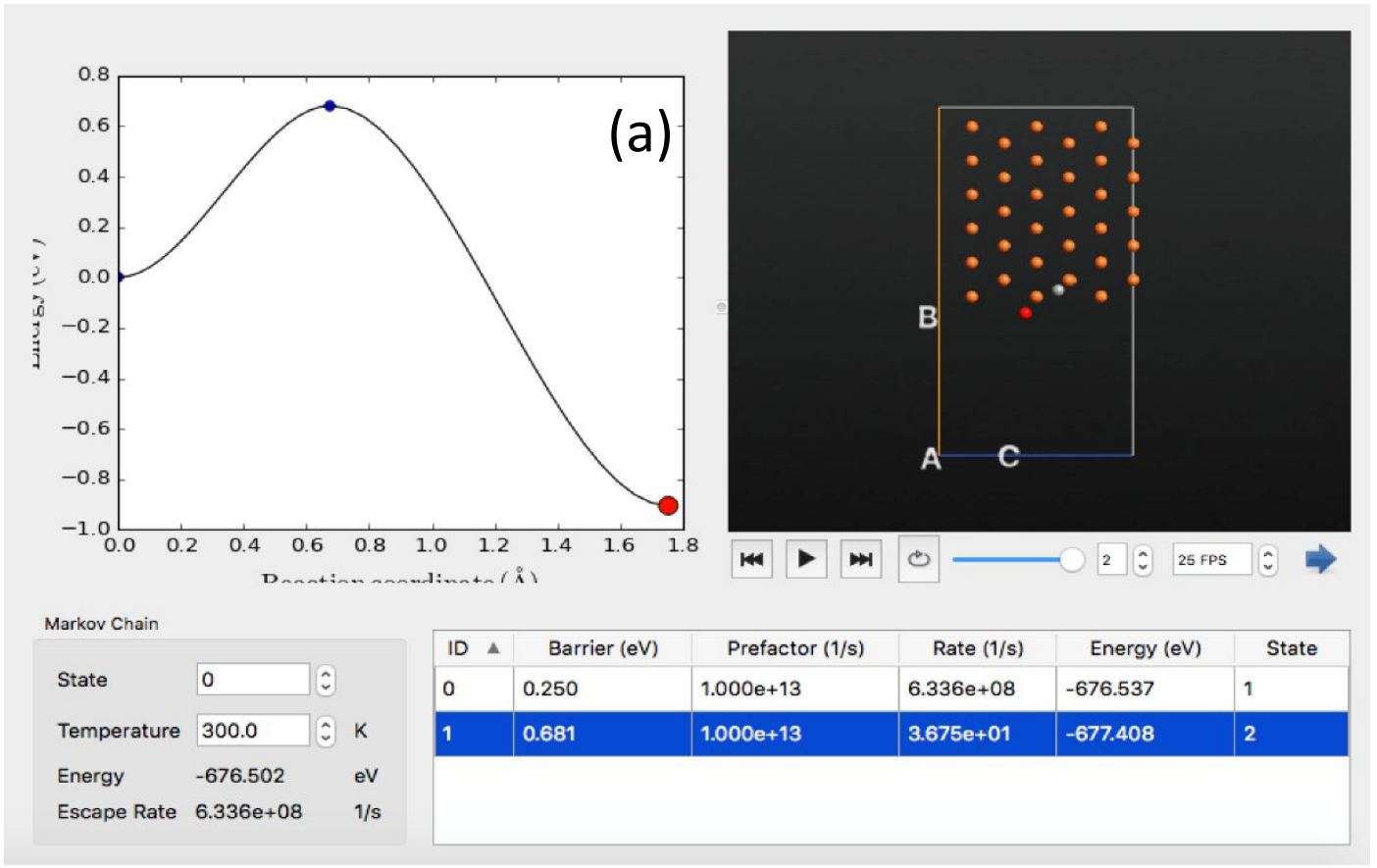

Figure S3: Adaptative Kinetic Monte Carlo (AKMC) simulations using extended ReaxFF within VNL-ATK software ${ }^{1,2}$ capturing the CO dissociation barrier on iron surface. 


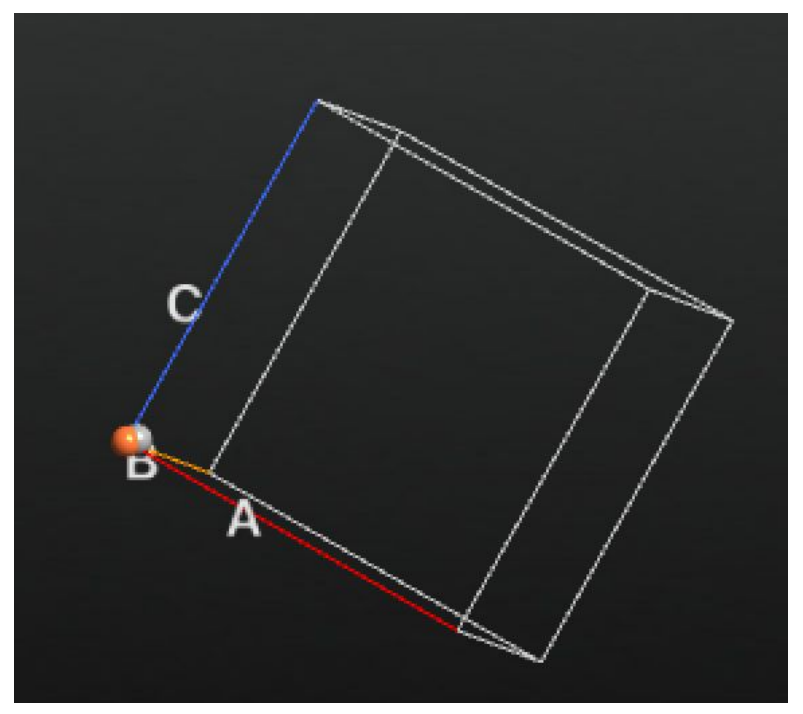

Figure S4: Ball and stick representation in a simulation box of $10 \times 10 \times 10 \AA^{3}$ of the unphysical very short Fe-C bond length of $0.3 \AA$, a known artifact of the RPOIC-2017 potential.

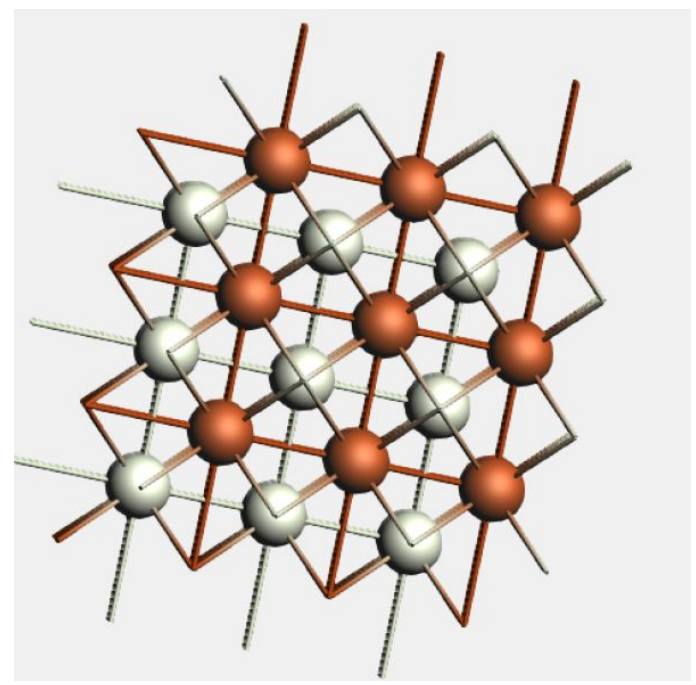

Figure S5: Ball and stick representation of bulk system distinguishing $\mathrm{Fe} \_\mathrm{B}$ (orange), $\mathrm{Fe} \_\mathrm{S}$ (white) tagged atoms after reparameterization of the "off diagonal" Fe-Fe parameters in extended ReaxFF. The lattice parameter is $2.87 \AA$, in excellent agreement with experimental reports ${ }^{3}$. 


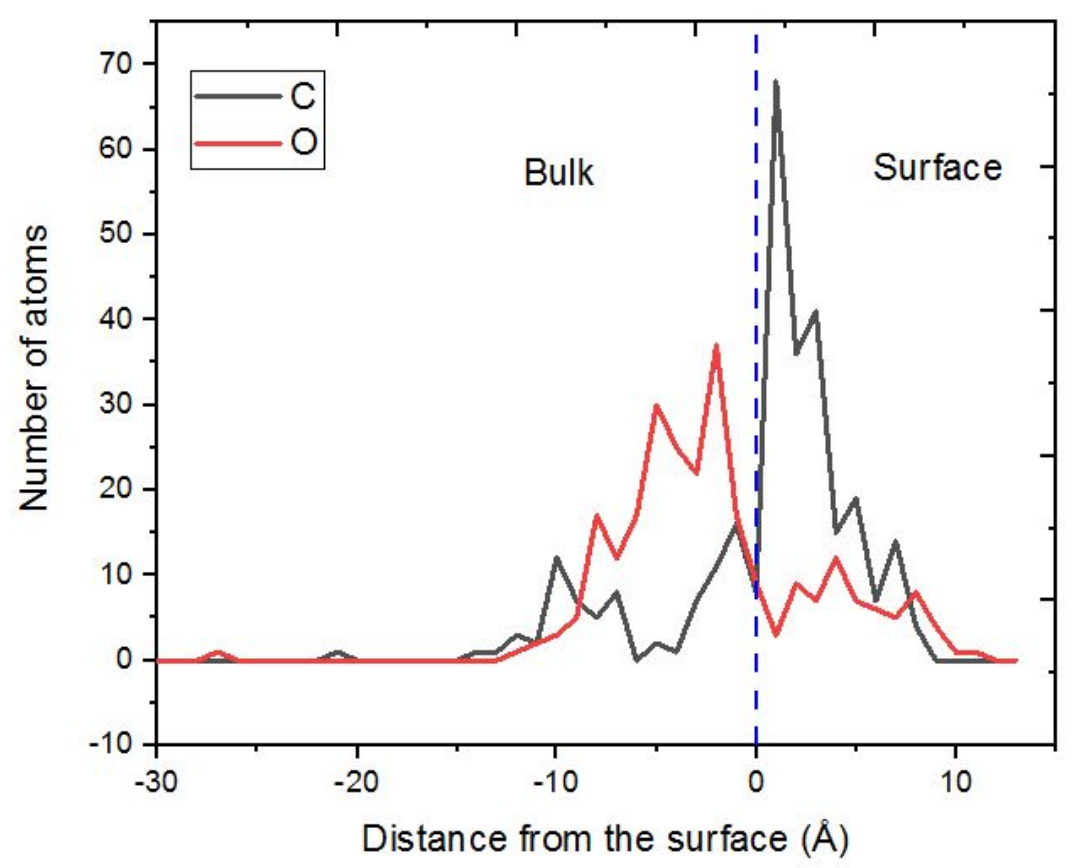

Figure S6: Linear distribution function of carbon (gray) oxygen (red) atoms.

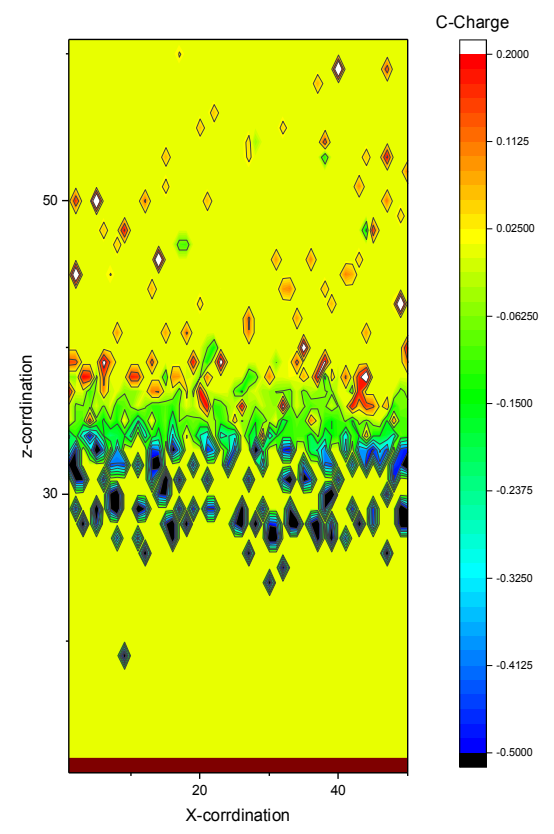

Figure S7: Charge map of carbon atoms. The carbon atoms are in different states (types of bonds): blue corresponds to highly coordinated states (C-Fe), red is low coordination states $(\mathrm{C}-\mathrm{O})$ and green corresponds to covalent states $(\mathrm{C}-\mathrm{C})$ bonds. 


\section{References :}

(1) Smidstrup, S.; Pedersen, A.; Stokbro, K.; Jónsson, H. Improved Initial Guess for Minimum Energy Path Calculations. J. Chem. Phys. 2014, 140 (21), 214106.

(2) Schneider, J.; Hamaekers, J.; Chill, S. T.; Smidstrup, S.; Bulin, J.; Thesen, R.; Blom, A.; Stokbro, K. ATK-ForceField: A New Generation Molecular Dynamics Software Package. Model. Simul. Mater. Sci. Eng. 2017, 25 (8), 85007.

(3) Sahputra, I. H.; Chakrabarty, A.; Restrepo, O.; Bouhali, O.; Mousseau, N.; Becquart, C. S.; El-Mellouhi, F. Carbon Adsorption on and Diffusion through the Fe (110) Surface and in Bulk: Developing a New Strategy for the Use of Empirical Potentials in Complex Material Set-ups. Phys. status solidi 2016. 